\title{
Bilateral Wilms' tumour: A ten-year experience of two academic centres in Johannesburg
}

\author{
Z J Solomon, ${ }^{1}$ FCPS (SA); A Withers, ${ }^{1} \mathrm{MB}$ ChB; T Govender, ${ }^{1}$ FCPS (SA); J Poole, ${ }^{2}$ FC Paed (SA); \\ R D Wainwright, ${ }^{2}$ DCH, FC Paed (SA); G Candy, ${ }^{3}$ MSc, PhD; J A Loveland, ${ }^{1}$ FCS (SA), Cert Paed Surg (SA) \\ ${ }^{1}$ Department of Paediatric Surgery, Chris Hani Baragwanath Academic Hospital and the School of Clinical Medicine, University of the \\ Witwatersrand, Johannesburg, South Africa \\ ${ }^{2}$ Department of Paediatric Oncology and Haematology, Charlotte Maxeke Johannesburg Academic Hospital and the School of Clinical Medicine, \\ University of the Witwatersrand, Johannesburg, South Africa \\ ${ }^{3}$ Research Department, Chris Hani Baragwanath Academic Hospital and the School of Clinical Medicine, University of the Witwatersrand, \\ Johannesburg, South Africa
}

Corresponding author: Z Solomon (zubrina.solomon@gmail.com)

\begin{abstract}
Background. Nephroblastoma is the most common paediatric renal malignancy, affecting 1 in 10000 children worldwide. Between 5\% and $10 \%$ present bilaterally.

Objective. To review two centre's experience of bilateral Wilms' tumour in order to improve future management practices.

Methods. This was a retrospective case review of nephroblastomas treated at the Chris Hani Baragwanath and Charlotte Maxeke academic hospitals from 1 January 2003 to 31 December 2013.

Results. Eighteen patients (8.04\%) presented with bilateral disease and were younger than those with unilateral disease. Three patients presented with metachronous disease at a median age of 23 months; initial presentation was at a median age of 2 months. The remaining 15 patients presented with synchronous disease at a median age of 27 months. Treatment followed aspects of the SIOP 9 protocol. Two patients died before surgery. Thirteen kidneys were removed. Twelve patients underwent nephron-sparing surgery, with microscopically positive resection margins seen in six kidneys post surgery. Two patients with residual microscopic disease relapsed. Three kidneys demonstrated unfavourable histology. Nephroblastomatosis was identified in one kidney. Eight patients were alive and disease free and three were alive with disease. An overall and disease-free survival rate of $66.67 \%$ and $55.56 \%$, respectively, was found for this cohort. Neither age $>2$ years nor metachronous disease was associated with a poorer prognosis.

Conclusion. Bilateral nephroblastoma is a complex disease. The majority of patients in this series presented with advanced local disease. Relapse was more commonly influenced by the presence of microscopically positive margins than metastatic disease at presentation.
\end{abstract}

S Afr J Child Health 2021;15(1):8-13. https://doi.org/10.7196/SAJCH.2021.v15.i1.1678

Wilms' tumour (WT), the most common renal malignancy in children, affects 1 in 10000 children worldwide, with 5 - 10\% of patients presenting with bilateral disease. ${ }^{[1-3]}$ Synchronous disease is defined as presentation with simultaneous bilateral tumours or presentation of a contralateral tumour within 2 months of the first. Approximately $1 \%$ of patients initially diagnosed with unilateral nephroblastoma will subsequently develop a lesion in the contralateral kidney (metachronous disease). ${ }^{[4,5]}$

Early diagnosis of bilateral Wilms' tumour (BWT) and follow-up of the contralateral kidney's condition in patients with unilateral disease are key to preserving renal function. ${ }^{[4]}$ The challenge in managing patients with BWT relates to balancing cure and preservation of renal function through nephron-sparing surgical techniques.

South Africa (SA) is classified as an upper middle-income country. ${ }^{[6]}$ Medical management and health infrastructure vary considerably across provinces, with infrastructure and resources in some regions being comparable with those in developed countries whereas lacking facilities in others prevents early referral and diagnosis. Without access to supportive care or the option of transplantation, the management of BWT is complicated, particularly as patients present late, which limits the treatment options available. ${ }^{[1,5]}$
Charlotte Maxeke Johannesburg Academic Hospital (CMJAH) and Chris Hani Baragwanath Academic Hospital (CHBAH) are two quaternary-care hospitals in Gauteng Province, SA. They serve an estimated population of 13.4 million, of whom 1.2 million are under 16 years of age. ${ }^{[7]}$ These are the only government-funded hospitals that offer paediatric surgery, including renal transplantation, and associated support facilities in the greater Johannesburg region. Patients presenting with paediatric nephroblastoma are managed by a multidisciplinary paediatric oncology team.

Following radiological identification of a renal mass, by either ultrasound or computerised tomography (CT), an ultrasoundguided percutaneous, retroperitoneal Tru-Cut biopsy or fine-needle aspiration is performed. Patients presenting with synchronous tumours are subsequently managed according to the International Society for Paediatric Oncology (SIOP) 9 protocol, which involves neoadjuvant chemotherapy followed by surgery; this is different from the management protocol earlier suggested by the Children's Oncology Group (COG), which involved initial surgery followed by adjuvant chemotherapy.

In the patient cohort reported on here, neoadjuvant chemotherapy was initiated with vincristine and actinomycin D. Following completion of the neoadjuvant protocol ( $4-8$ weeks on average in the majority of patients), tumours were re-imaged by CT to assess 
response. If poor or no chemotherapeutic response was observed, a further five cycles of chemotherapy were initiated, with the addition of adriamycin, followed by nephron-sparing surgery.

Management of patients with BWT aims to provide effective curative treatment while preserving sufficient functioning renal parenchyma. ${ }^{[4,5]}$ Management of metachronous disease is the same as for patients with unilateral nephroblastoma.

The present study describes our experience with treating patients with BWT over the period 2003 - 2013 and the observed outcomes, and compares findings with those reported by other institutions. ${ }^{[4,5]}$

\section{Methods}

The study included case files of all children aged 0 - 16 years and who presented with BWT to the paediatric oncology units at the CMJAH and CHBAH from 1 January 2003 to 31 December 2013. Institutional approval for reviewing the files of this period was obtained from the Human Ethics Research Committee of the University of the Witwatersrand (ref. no. M140629). Patient demographics, diagnosis, histological subtype of the tumour, neoadjuvant treatment, surgical intervention (including timing of surgery and surgical technique), postoperative management and outcome (survival, renal function and complications) were recorded.

Statistical analyses were performed using Statistica (version 13) (Tibco Software Inc., USA). Patient age is expressed as medians and the associated interquartile range (IQR). Analysis of overall and disease-free survival was performed using Stata (version 14.2, StataCorp., USA), with glomerular filtration rate (GFR) calculated according to the modified Schwartz formula.

\section{Results}

A total of 224 patients presented with WT, at a median (IQR) age of 39.5 ( 1 - 172) months. Of these, 18 patients $(8.04 \%)$ were diagnosed with BWT, at a median (IQR) age of $30(9-145)$ months. Three of the 18 patients $(16.60 \%)$ presented with metachronous disease at a median (IQR) age of $44(25$ - 144) months; initial tumour presentation was at a median age of 2 months (range 2 - 31 months) (Table 1).

One patient was found to have had Beckwith-Wiedemann syndrome and hemi-hypertrophy, with hypermethylation in the H19 differentially hypermethylated region on chromosome $11 \mathrm{p} 15$. This was found to be a familial condition, as his mother presented with a unilateral WT at 3 years of age. His management involved bilateral nephron-sparing surgery, which allowed $90 \%$ of the left kidney and $40 \%$ of the right kidney to be preserved. Histological assessment demonstrated positive resection margins in the left nephrectomy specimen, which showed epithelial-predominant (intermediate) histology. This patient was well and disease free at last follow-up (92 months post diagnosis).

The median (IQR) age for presentation of synchronous disease was $27(9-60)$ months. Characteristics of the presentation, management and outcomes of cases are described in Table 2. The majority of patients presented with stage 3 disease, as determined from CT imaging.

Three patients (16.67\%) presented with discordant histology, of whom two had anaplastic histology. One of the patients with anaplastic histology has since died from the initial disease process and the other was alive and free of disease, without history of relapse, at the last follow-up.

Both patients who presented with stage 4 disease (lung metastasis) were treated with a high-risk chemotherapeutic protocol and lung radiation. One of these patients received a total nephrectomy and partial nephrectomy that showed triphasic histology. This patient was still alive and free of disease at last follow-up. The other had anaplastic histology and died before a staged partial nephrectomy could be performed. None of the histology specimens taken from the cohort demonstrated nephroblastomatosis in isolation. One patient with synchronous BWT had teratoid histology diagnosed through bilateral open biopsies.

Fifteen in-theatre operative procedures were performed (Table 3). Four patients presented with caval thrombus, but only one required renal vein exploration and caval thrombectomy following neoadjuvant chemotherapy. The patient with bilateral teratoid histology developed intraoperative tumour rupture.

Of the patients who had been identified for further surgery post initial nephrectomy, one died before further surgery, one refused further surgery and one kidney had no radiological or clinical evidence of disease after neoadjuvant chemotherapy, having initially had a positive diagnosis on biopsy. Both of the latter two patients were alive at last follow-up. Histology revealed positive microscopic margins in six patients who had nephron-sparing surgery. Two of these patients (33.30\%) relapsed outside the renal bed; however, this relapse was not statistically significant $(p=0.78)$.

One patient developed a urinoma 1 month after right partial nephrectomy. A double J-stent was inserted and removed a month later.

Kaplan-Meier curves (Figs 1 and 2) demonstrate overall and event-free survival as $66.67 \%(n=12 / 18)$ and $55.56 \%(n=10 / 18)$, respectively, over the 10 -year period. The six mortalities were due to cardiac failure associated with adriamycin, 27 months after initiation $(n=1)$, sepsis $(n=2)$ and metastatic disease progression $(n=3)$. There was a low level of recurrence of disease for patients older than 24 months. Only one of the three patients with metastatic disease at relapse died.

Two patients developed renal failure. Their GFR was $11.6 \mathrm{~mL} /$ min and $9.7 \mathrm{~mL} / \mathrm{min}$ per $1.73 \mathrm{~m}^{2}$, respectively. This presented in the immediate postoperative period, secondary to overwhelming sepsis and subsequent multiorgan failure. Neither patient survived long enough to receive renal replacement therapy. The majority of patients (57\%) maintained a normal GFR and 29\% had mild renal dysfunction when GFR was calculated (range 11.6 - 128.4 $\mathrm{mL} / \mathrm{min}$ per $1.73 \mathrm{~m}^{2}$ ). In patients with mild renal dysfunction (all of whom had nephrectomy or partial nephrectomy), at least $50 \%$ of the renal parenchyma of the kidney that underwent nephrectomy was left in situ. Only one of these patients was on chronic antihypertensive medication postoperatively, having been normotensive preoperatively.

\section{Discussion}

Similar to other studies, we found the most common presenting symptom of BWT to be an abdominal mass. ${ }^{[1,4,5,8]}$ Literature suggests synchronous disease accounts for 4 - 7\% of all cases of WT presenting at a younger age than unilateral disease, ${ }^{[8,9]}$ as was also observed from the data in our series.

Our series showed a smaller percentage of patients with metachronous disease (17.60\%) than has been reported elsewhere in the literature (35\%). ${ }^{[10]}$ A patient who presented with hypermethylation of the differentially methylated region of H19 on chromosome 11p15 in our series, seen as a prognostic factor associated with relapse, ${ }^{[11]}$ has remained disease free.

The published incidence of metastasis is $\sim 10 \% .{ }^{[12]}$ In our series, $28 \%$ of the patients $(n=5 / 18)$ developed metastatic disease. Although $11 \%$ of patients $(n=2)$ presented with metastatic disease on first examination, advanced local disease was found in the majority of patients on presentation. In a paper assessing the challenges to treatment in sub-Saharan Africa, it has been noted that patients often 


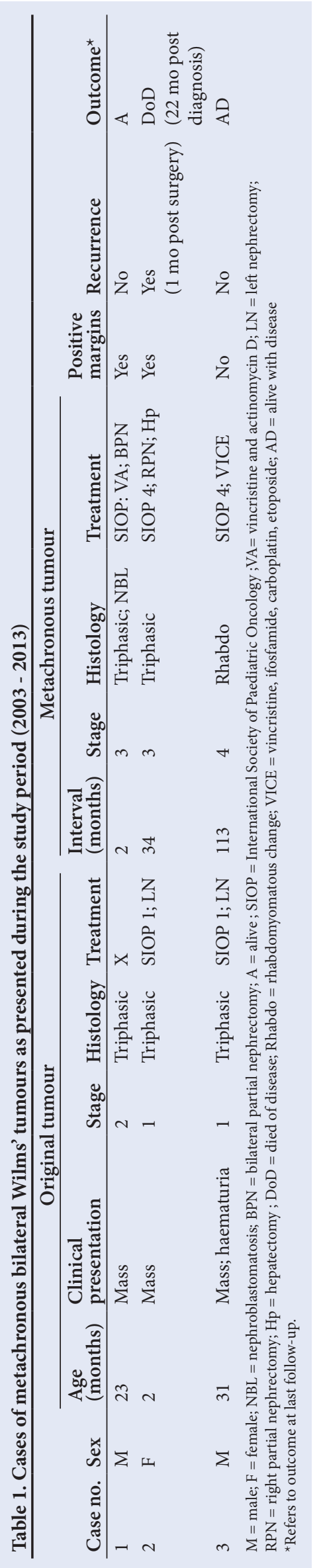

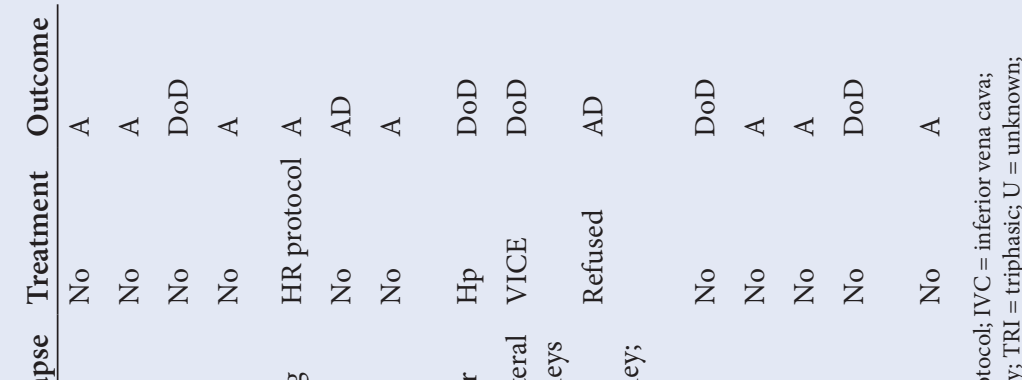

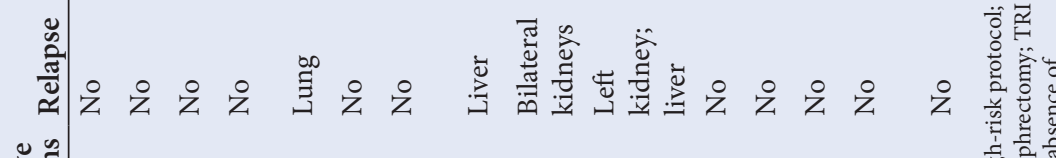

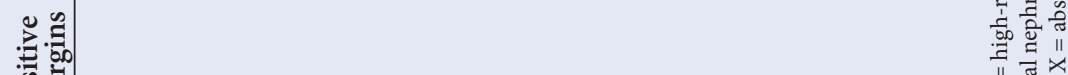

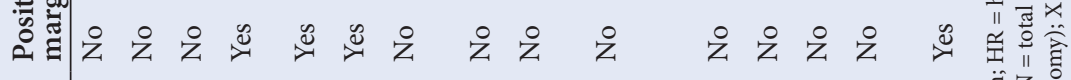

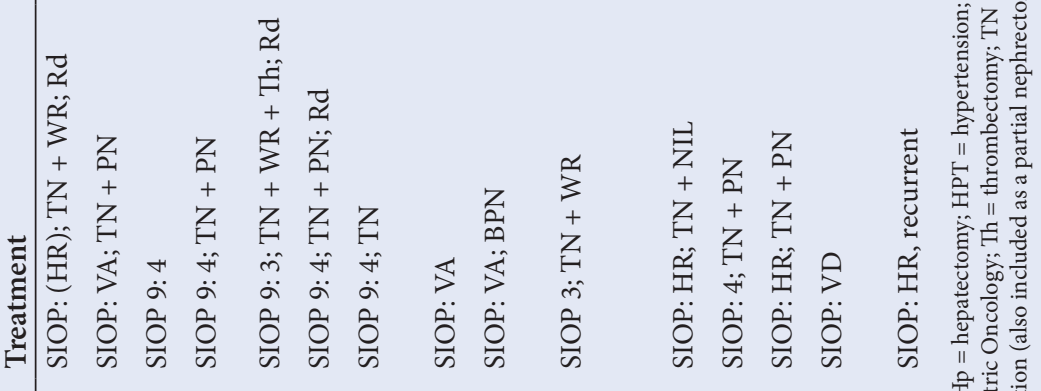

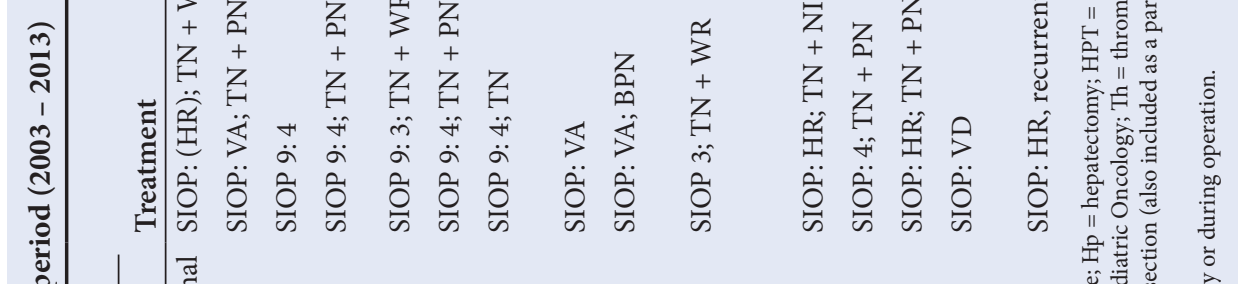

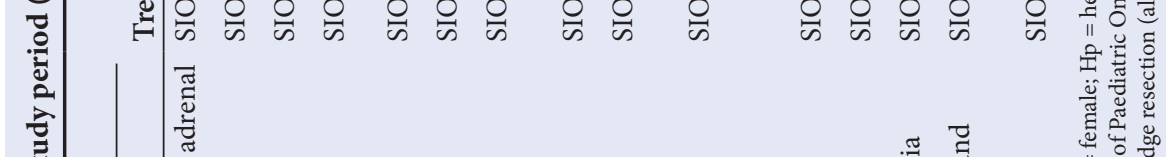

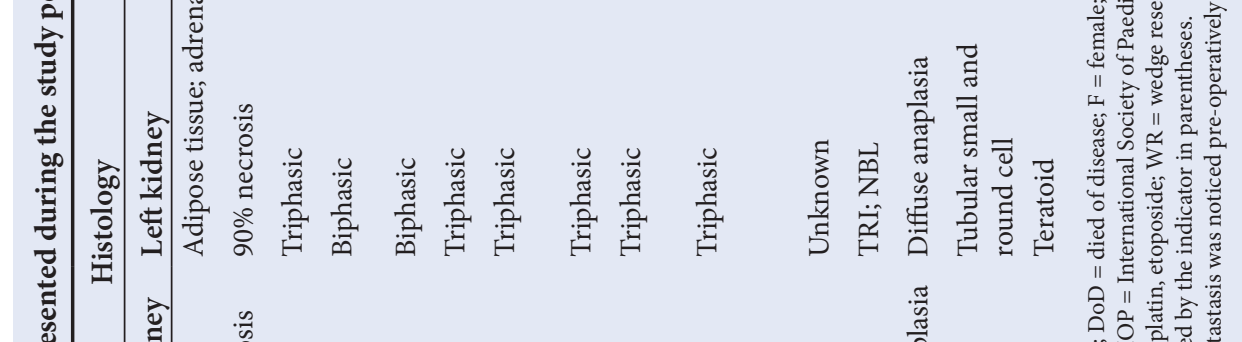

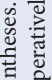

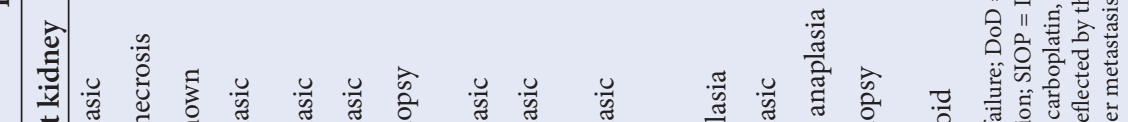

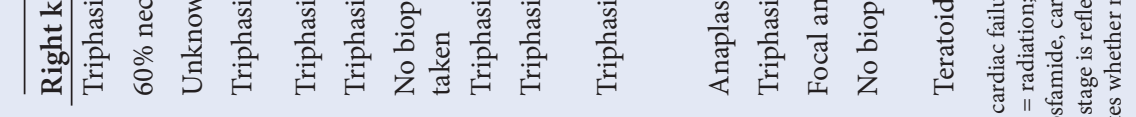
UE $\bar{z} \quad \bar{z}$

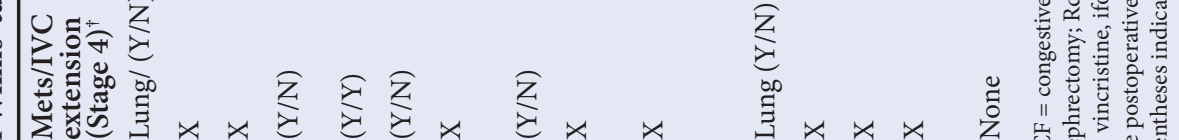

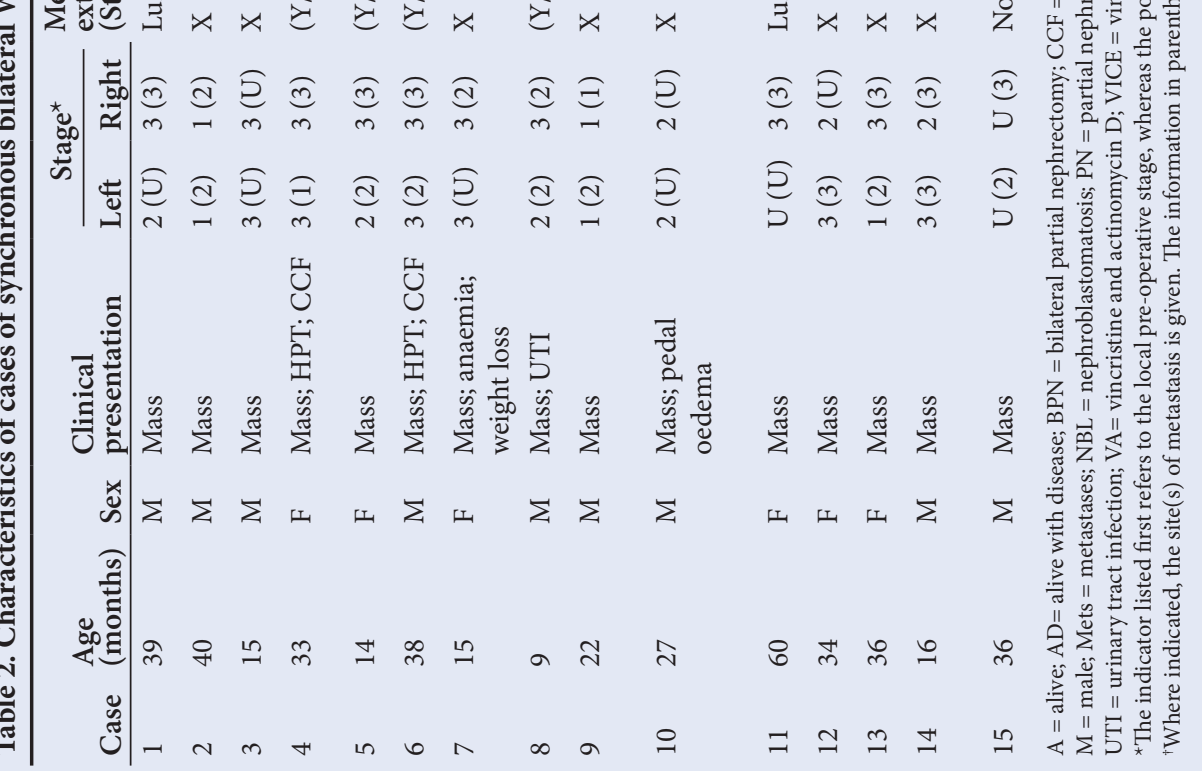


Table 3. Summary of surgical procedures performed during the study period $(2003-2013)$

\begin{tabular}{lll}
\hline Index kidney & Contralateral & $N=18, n(\%)^{*}$ \\
\hline Nephrectomy & Wedge resection & $5(27.8)$ \\
Partial nephrectomy & Partial nephrectomy & $3(16.7)$ \\
Nephrectomy & Partial nephrectomy & $4(22.2)$ \\
Nephrectomy & No surgery & $3(16.7)$ \\
Needle biopsy & Needle biopsy & $1(5.5)$ \\
Needle biopsy & No surgery & $1(5.5)$
\end{tabular}

${ }^{*} \mathrm{~A}$ total of 18 patients were included in the series, but only 15 operative procedures were performed. The two needle biopsies are included only for reference.

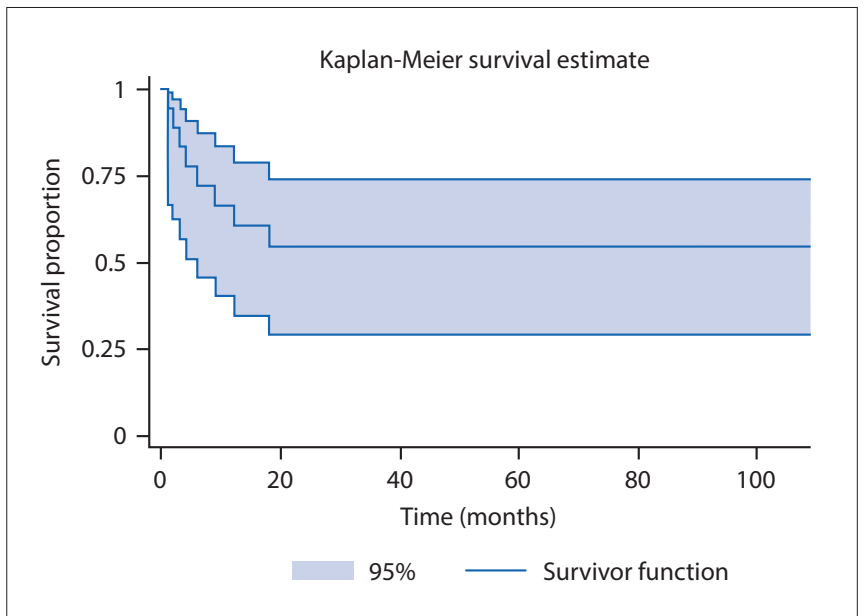

Fig. 1. Event-free survival of patients with bilateral Wilms' tumour treated during the study period (2003 - 2013) (CI = confidence interval.)

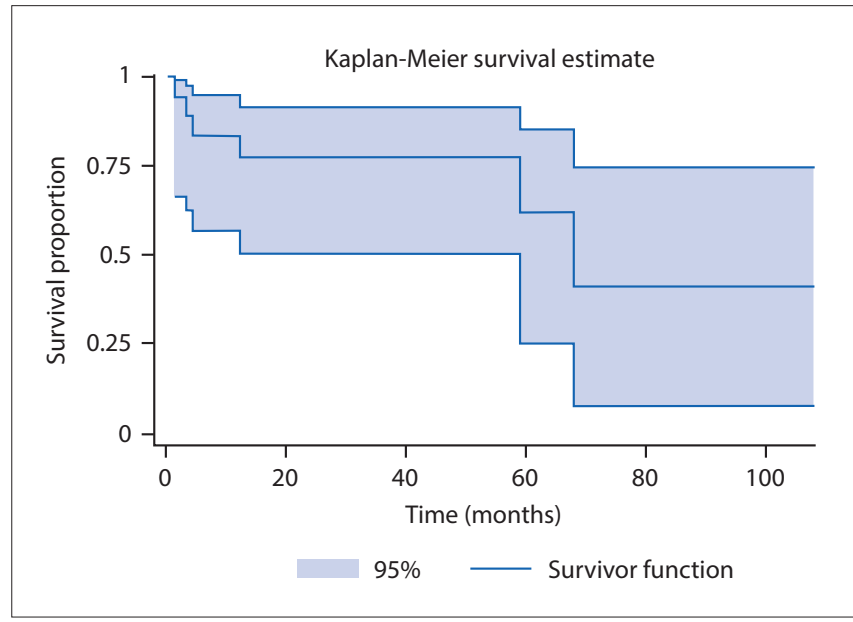

Fig. 2. Overall survival estimate of patients with bilateral Wilms' tumour treated during the study period (2003 - 2013.)

present with more advanced disease and associated malnutrition. ${ }^{[13]}$

The typical triphasic histological structure was the most common histological finding in our series, concordant with current literature. ${ }^{[14]}$ According to the SIOP guidelines, patients with WT can be stratified as having low, intermediate or high risk based on histology results. ${ }^{[15]}$ Such risk stratification, together with postoperative locoregional and systemic stage, informs treatment decisions. Accordingly, the majority of our patients (67\%) were classified as having intermediate risk and $33 \%$ were classified as high risk. None of the patients were classified as having low risk.
All patients were treated according to their risk stratification. In keeping with the literature, a small number of histological specimens $(n=2 ; 11 \%)$ were anaplastic, which may predict a poor chemotherapeutic response. ${ }^{[4,5,8]}$ Anaplasia may be focal or diffuse, with focal anaplasia being associated with a better outcome..$^{[4,8,15]}$ Discordant histology can be present in up to $20 \%$ of cases. ${ }^{[16]}$

Nephrogenic rests (nephroblastomatosis) are precursor lesions of WT. ${ }^{[17]}$ They are seen in up to $90 \%$ of synchronous and $94 \%$ of metachronous lesions. ${ }^{[18]}$ However, in our series, only one patient displayed nephrogenic rests in the initial histological specimens (but without developing recurrence). This unusual histological presentation may be related to pathology reporting not being standardised. Without dedicated expert review, nephroblastomatosis may have been missed. Nephroblastomatosis presents a diagnostic challenge as it can appear radiologically and histologically similar to WT. ${ }^{[14]}$ Close monitoring for conversion to WT in patients who have this histological finding is warranted. ${ }^{[5]}$

In a prospective multi-institutional study by the COG in 2015, patients were treated with neoadjuvant chemotherapy for $6-12$ weeks before nephron-sparing surgery. ${ }^{[18]}$ This protocol showed improved early outcomes and is now the COG's preferred practice for BWT. ${ }^{[19]}$

The preservation of renal function is more challenging in patients presenting with advanced disease resistance to chemotherapy or in patients with metachronous WT. ${ }^{[4,5]}$ Surgical decision-making is critical and deciding whether to operate on both kidneys simultaneously or rather staging the surgery should be carefully considered. The current recommendation is to operate on the kidney least affected by the disease process. ${ }^{[17]}$

Techniques for nephron-sparing surgery are varied and innovative ${ }^{[20]}$ At a tertiary hospital in Cape Town, $\mathrm{SA},{ }^{[4]}$ techniques have moved away from definitive control of the renal hilum and now rather focus on the use of topical cooling of the kidney coupled with the use of diathermy or an ultrasonic scalpel to achieve good outcomes. Surgeons in a tertiary-level institute in KwaZulu-Natal provnice, in turn, consider isolation of the renal vasculature at the hilum to be an important part of the operation. ${ }^{[5]}$ Davidoff et al. ${ }^{[21]}$ have described manual compression of vessels post identification, before the parenchyma is oversewn with absorbable suture. The exposed renal parenchyma may be coagulated with an argon beam to further ensure haemostasis. ${ }^{[22]}$ In our group, we excise the tumour utilising monopolar diathermy. This allows excellent haemostasis and visualisation when required to close the calyceal system, while avoiding the need for vascular control or induced hypothermia. Regardless of the technique used, it is important to avoid traction or torsion of the arteries so as to prevent spasm, intimal damage and vascular occlusion. ${ }^{[21]}$

Complications of surgical management of BWT are generally minor and infrequent. In our group, we aim to resect all gross macroscopic tumour, with the knowledge that residual microscopically positive margins may remain. We have found that this disease is adequately treated with adjuvant chemotherapy. In our cohort, relapse occurred in two of the six kidneys that had positive microscopic margins on histology post nephron-sparing surgery. Several reports have suggested that overall survival for patients with positive margins is not negatively affected by surgery and that the most important factors for relapse are histology type and the presence of metastases. Bilateral nephron-sparing surgery is often possible despite the large size of some tumours and should be attempted despite the possibility of microscopically positive margins. ${ }^{[21,22]}$ Should resection leave insufficient nephron mass and cause renal failure, renal transplantation remains an 
option; however, few patients in our cohort came to need renal replacement.

With advancing treatment regimens, the outcome of renal function in patients with BWT is improving. ${ }^{[4,9,11,15,20,21,23]}$ However, with varying follow-up times, no conclusions regarding long-term risks of failure into adulthood can be made. Recently, a 15-year follow-up study found that the long-term risk of renal failure was $15 \% .{ }^{[24]}$ Renal dysfunction in our series was infrequent and mild in patients who did develop it, therefore validating the decision to forego the use of renal replacement lines in these patients in the postoperative period. As these functions are calculated in the intermediate period, they may well worsen secondary to the effect of nephrotoxic drugs in the long term. Many centres avoid abdominal radiation in patients who have received nephronsparing surgery because of the associated renal toxicity and risk of secondary malignancy. ${ }^{[16]}$ In our series, three patients with inferior vena caval tumour thrombosis who survived to surgery received post-surgical radiation therapy to the entire abdomen. There was no mention in the clinical files of whether the residual kidney was shielded during radiation. Such patients' renal function and development of secondary malignancies will require long-term follow-up.

Disease-free and overall survivals in BWT cases have improved. ${ }^{[3,8,15,22]}$ SA studies have reported encouraging survival rates, ranging from $70 \%$ to $85 \%$ at varying times of follow-up. ${ }^{[4,5]}$ The survival curves in our study demonstrate that most individuals died early in the disease process, either from advanced disease or from complications of treatment. Deaths that occurred after a latent period were attributed to relapse. Our findings show lower overall and disease-free survival rates $(66.67 \%$ and $55.56 \%$, respectively) compared with previously mentioned studies at the time of last follow-up (follow-up period extended to 9 years).

The cohort in the study by Hadley et al ${ }^{[5]}$ had a higher survival rate. They followed a similar SIOP protocol to that used in our unit, but had a slightly bigger cohort $(n=20)$; a comparison with their findings is therefore apt. In that study, more tumours $>1000 \mathrm{~g}$ were operated on and a higher rate of discordant histology (56\%) was reported than in our study. Only four of their patients had visceral metastases at presentation: two to both lung and liver, two to lung only. Three patients presented with rupture. The longer the followup of patients, the more likely it will be to detect deaths secondary to progression of the disease and which occur more than 2 years after treatment (deaths associated with adverse treatment and tumourrelated events).

\section{Study limitations}

This was a retrospective study with a small sample size, given the rarity of the disease entity. This made a comparative study of the various surgical techniques difficult and prevented definitive associative conclusions about the recurrence, long-term outcomes, survival and adjuvant medical management on survival. Two patients were lost to follow-up, which has implications for the general conclusions of the study as it is difficult to assess their contribution in this relatively small series. In addition, nonstandardised methods of pathology reporting, skill level of the pathologist and a non-dedicated histology team could have negatively affected the results.

\section{Recommendations}

A further prospective study regarding the management of our patients according to the current SIOP protocols would provide more information to help improve management of BWT patients, with an opportunity to intervene more readily at specific points of management. Follow-up of the current group of patients with regard to renal function outcomes could give insight into the postoperative course over the long term, as mild deterioration may either remain static or worsen progressively, which cannot be predicted from the current data and time of follow-up. We are involved in a comparative study between our cohort of patients, and similar cohorts in the UK, New Zealand and Nigeria. A large collaborative study like this may yield further insights into the management of BWT patients. However, to truly provide insight into our management of this disease entity, given the SA patient profile, a combined study between paediatric surgical units that manage oncology patients should be performed. Radionucleotide GFR determination postoperatively appears to be the way forward for appropriate monitoring of renal function.

\section{Conclusion}

The majority of patients with synchronous disease (73\%) presented late and with a higher rate of local-stage disease. Patients who received surgery rarely required renal replacement therapy or transplantation (required only in $11 \%$ of cases owing to sepsis-related renal failure). It is advised that this group of patients should continue to be followed up to assess the need for potential renal replacement therapy or transplantation in the future and to identify recurrence of disease. Nephroblastomatosis was a rare finding in our series (found only in one patient). The presence of microscopically positive margins on histology specimens (50\% of partial nephrectomy specimens) was not a significant predictor of recurrent disease. Although two of these patients relapsed, it did not translate to a worse overall survival. Recurrence, when located outside the renal bed, predicted poor survival. Despite late presentation, fair overall and event-free survival was achieved (66.7\% and 55.6\%, respectively) with neoadjuvant chemotherapy and nephron-sparing surgery. Other tertiary-level hospitals in SA that deal with late-presenting patients have shown better survival rates at shorter periods of followup. However, the main factor affecting outcomes in our group when compared with findings from other SA centres (who also manage patients according to the SIOP protocol) is the higher proportion of late-stage patients with advanced local disease.

\section{Declaration. None.}

Acknowledgements. We thank Drs A Grieve and DS Harrison for their advice during drafting of the manuscript.

Author contributions. ZJS was the principal investigator and primary author of the manuscript, supported by TG and JAL. The remaining authors all contributed to refining the manuscript and statistical analyses. AW was responsible for generating the Kaplan-Meier curves and gave input regarding interpretation of the statistical analysis.

Funding. None.

Conflicts of interest. None.

1. Seyed-Ahadi M-M, Khaleghnejad-Tabari A, Mirshemirani A, Sadeghian N Amonnollahi O. Wilms' Tumor: A 10 year retrospective study. Arch Iranian Med 2007;10(1):65-69.

2. Farhat W, McLorie G, Capolicchio G. Wilms' tumor. Surgical considerations and controversies. Urol Clin North Am 2000;27(3):455-461. https://doi. org/10.1016/s0094-0143(05)70093-8

3. Hamilton TE, Ritchey ML, Haase GM, et al. The management of synchronous bilateral Wilms tumor: A report from the National Wilms Study Group. Ann Surg 2011;253(5):1004-1010.

4. Millar JW, Davidson A, Rode H, et al. Bilateral Wilms' tumors: A singlecenter experience with 19 cases. J Pediatr Surg 2005;40:1289-1294. https://doi org/10.1016/j.jpedsurg.2005.05.013 
5. Hadley GP, Mars M, Ramdial PK. Bilateral Wilms' tumour in a developing country: A descriptive study. Pediatr Surg Int 2013;29(4):419-423. https://doi. org/10.1007/s00383-013-3287-7

6. Dell A, Numanoglo A, Arnold M, Rode H. Pediatric surgeon density in South Africa. J Pediatr Surg 2018;53(10):2065-2071. https://doi.org/10.1016/j. jpedsurg.2017.11.067

7. Statistics South Africa. Community survey 2016. Statistical release. Pretoria: SSA, 2016.. http://cs2016.statssa.gov.za/wp-content/uploads/2016/07/NT-3006-2016-RELEASE-for-CS-2016-_Statistical-releas_1-July-2016.pdf (accessed 2 September 2017).

8. Neville HN, Ritchey ML. Wilms' tumor. Overview of National Wilms' Tumor Study Group results. Urol Clin North Am 2000;27:435-442. https://doi. org/10.1016/s0094-0143(05)70091-4

9. Tomlinson GS, Cole CH, Smith NM. Bilateral Wilms' tumor: A clinicopathologic review. Pathology 1999;31:12-16. https://doi.org/10.1080/003130299105458.

10. Desai D, Nicholls G, Duffy PG. Bench surgery with autotransplantation for bilateral synchronous Wilms' tumor: A report of three cases. J Pediatr Surg 1999;34(4):632-634. https://doi.org/10.1016/s0022-3468(99)90092-1

11. Fernandes CV, Perlman EJ, Mullen EA, et al. Clinical outcome and biological predictors of relapse after nephrectomy only for very low-risk Wilms tumor: A report from Children's Oncology Group AREN0532. Ann Surg 2017;265(4):835-840. https://doi.org/10.1097/SLA.0000000000001716

12. Elashry R. Bilateral Wilms' Tumor: Mansoura multi-centers 15 years experience. J Oncol Pharm Pract 2012;18(1):115-121. https://doi. org/10.1177/1078155210396575

13. Isreals T. Wilms tumor in Africa: Challenges to cure. Pediatr Blood Cancer 2012;58(1):3-4.

14. Indolifi $\mathrm{P}$ Jenker A, Terenziani $\mathrm{M}$, et al Synchronous bilateral Wilms tumor: A report from the Associazione Italiana Ematologia Oncologia Pedriatica(AIEOP). Cancer 2013;119:1586-1592. https://doi.org/10.1002/cncr.27897

15. Dome JS, Perlman EJ, Graf N. Risk stratification for Wilms tumor: Current approach and future directions. Am Soc Clin Oncol Educ Book 2014:215-223.
16. Millar AJW, Cox S, Davidson A. Management of bilateral Wilms tumours. In: Van den Heuvel-Eibrink MM, ed. Wilms tumour. Brisbane: Codon Publications, 2016:61-74. https://doi.org/10.15586/codon.wt.2016.chapter5

17. Coppes MJ, Pritchard-Jones K. Principles of Wilms' tumor biology. Urol Clin North Am 2000;27(3):423-433. https://doi.org/10.1016/s0094-0143(05)70090-2

18. Millar AJW, Davidson A, Rode H, Numanoglu A, Hartley PS, Desai F. Nephronsparing surgery for bilateral Wilms' tumours: A single-centre experience with 23 cases. Afr J Paediatr Surg 2011;8(1):49-56. https://doi.org/10.4103/01896725.78669

19. Ehrlich PF, Chi Y, Chintagumpala MM, et al. Results of the first Prospective Multi-Institutional Treatment Study in Children with Bilateral Wilms Tumor (AREN0534): A report from the Children's Oncology Group. Ann Surg 2017;266(3):470-478. https://doi.org/10.1097/SLA.0000000000002356

20. Kieran K, Davidoff AM. Nephron-sparing surgery for bilateral Wilms tumor Pediatr Surg Int 2015;31(3):229-236. https://doi.org/10.1007/s00383-015-3668-1

21. Davidoff AM, Giel DW, Jones DP, et al. The feasibility and outcome of nephronsparing surgery for children with bilateral Wilms tumor. The St Jude Children's Research Hospital experience: 1999-2006. Cancer 2008;112(9):2060-2070. https://doi.org/10.1002/cncr.23406

22. Hamilton TE, Ritchey ML, Haase GM, et al. The management of synchronous bilateral Wilms tumor: A report from the National Wilms Study Group. Ann Surg 2011;253(5):1004-1010. https://doi.org/10.1097/SLA.0b013e31821266a0

23. Kubiak R, Gundeti M, Duffy PG, Ransley PG, Wilcox T. Renal function and outcome following salvage surgery for bilateral Wilms' tumor. J Pediatr Surg 2004;39(11):1667-1672. https://doi.org/10.1016/j.jpedsurg.2004.07.009

24. Cooper CS, Jaffe WI, Huff DS, et al. The role of renal salvage procedures for bilateral Wilms tumor: A 15-year review. J Urol 2000;163:265-268.

Accepted 16 September 2020. 